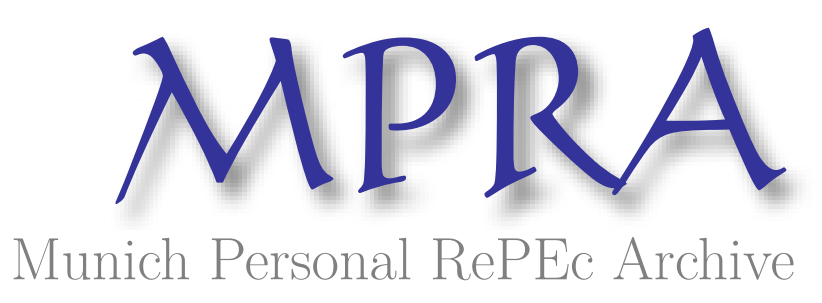

\title{
Duration of Business Cycles
}

Everts, Martin

University of Bern

April 2006

Online at https://mpra.ub.uni-muenchen.de/1219/

MPRA Paper No. 1219, posted 20 Dec 2006 UTC 


\title{
Duration of Business Cycles
}

\author{
Martin P. Everts*
}

April 2006

\begin{abstract}
In this paper the Bry and Boschan (1971) procedure is modified such that it can be applied to quarterly data in order to recalculate the maximum duration of business cycles. In this way it can be shown that the maximum duration of business cycles constitutes 42 quarters in the United States of America and 49 quarters in the United Kingdom. The large difference to the maximum duration of Burns and Mitchell (1946) makes clear that caution is advisable with the application of the filters by Baxter and King (1999) and Christiano and Fitzgerald (2003). If one chooses the maximum duration too low (high), the amplitude of the medium-term business cycles is underestimated (overestimated) and the variability of the growth rate of the long-term trend is overestimated (underestimated).
\end{abstract}

JEL Classification: E32, E37

\footnotetext{
${ }^{*}$ University of Bern, Department of Economics, Schanzeneckstrasse 1, CH-3001 Bern, Switzerland, Tel: +41 (0)31 63180 76, E-Mail: martin.everts@vwi.unibe.ch. I would like to thank Ernst Baltensperger, Andreas Fischer, Roland Holder, Robert King, Simon Lörtscher, Klaus Neusser and Frank Schmid, as well as seminar participants at the Swiss Society of Economics and Statistics Annual Meeting in Lugano and Spring Meeting of Young Economists in Seville for helpful comments and support.
} 


\section{Introduction}

Whoever deals with business cycles will hear the names of Burns and Mitchell. Mitchell (1927, p. 468) for example provided an often cited definition: ${ }^{1}$

Business cycles are a species of fluctuations in the economic activities of organized communities. The adjective business restricts the concept to fluctuations in activities which are systematically conducted on a commercial basis. The noun cycles bars out fluctuations which do not recur with a measure of regularity.

Unfortunately, Burns and Mitchell are not only cited for their definitions of business cycles but rather often also in connection with their basic properties. Last but not least, the book of Burns and Mitchell is also consulted as a reference for the minimum and maximum duration of business cycles.

Over the course of the last few years different new methods to measure business cycles were developed. Especially the methods from the field of spectral analysis obtained great popularity, among other things because they get by with very few assumptions on the business cycle. One of the assumptions which has to be made concerns the minimum and maximum duration of a business cycle. For example, when applying the high-pass filter methods by Prescott (1986) and King and Rebelo (1993) a minimum duration has to be defined, whereas the band-pass filters by Stock and Watson (1998), Baxter and King (1999) and Christiano and Fitzgerald (2003) require a minimum and a maximum duration.

When indicating the minimum and maximum duration, most authors refer, directly or indirectly, to the calculations of Burns and Mitchell (1946). This raises a problem, as Burns and Mitchell calculated their figures on a data series ranging from 1885 to 1931 . With other words, the figures for the minimum and maximum duration that are nowadays used by many authors are based on very old data. This is astonishing for mainly two reasons: Firstly, the National Bureau of Economic Research (NBER) regularly publishes new and updated

\footnotetext{
${ }^{1}$ Burns and Mitchell (1946, p. 3) provided a very similar, likewise often cited definition a few years later.
} 
data for the minimum and maximum duration of the business cycles in the United State of America; however there is no known article which utilizes this data. Secondly, already Burns and Mitchell noticed that the data concerning the characteristics of business cycles changes over the course of the years.

The following chapters attend to the minimum and maximum duration of business cycles. Chapter 2 briefly highlights the minimum and maximum duration calculated by Burns and Mitchell (1946) as well as different methods to identify the characteristics of business cycles. In chapter 3 the method of Bry and Boschan (1971) is modified in a way such that it is applicable to quarterly data. This procedure is then verified in chapter 4 using business cycle data of the United States of America. This verification shows that the procedure developed in chapter 3 delivers very exact data about the characteristics of business cycles, and thus, it is qualified to examine the characteristics of business cycles of other countries. As several authors have already studied the characteristics of business cycles of different countries, chapter 6 and 7 focus on the analysis of business cycles of the sectors and industries of the United Kingdom. Finally, chapter 8 clarifies whether the changed data of the minimal and maximal duration of business cycles have an impact on the filters developed by Baxter and King (1999) and Christiano and Fitzgerald (2003), and could result in misleading conclusions about the assessment of business cycles.

\section{Duration of Business Cycles}

Burns and Mitchell (1946) define the duration of a business cycle for the United States of America between a minimum of 16 and 22 months and a maximum of 100 and 106 months. For the United Kingdom the values lie between minimal 16 and 22 months and maximal 135 and 141 months. In quarters this would be minimal 5 to 7 quarters and maximal 33 to 35 quarters in the United States, and 45 to 47 quarters in the United Kingdom.

As mentioned before, Burns and Mitchell (1946) are often cited in the lit- 
erature - albeit with varying accuracy and reliability. Baxter and King (1999) refer to Burns and Mitchell, but declare the minimum and maximum duration of a business cycle 6 respectively 32 quarters. Other studies which likewise refer to Burns and Mitchell are among others King and Plosser (1994), Stock and Watson (1998) or Christiano and Fitzgerald (2003).

It is astounding that the data from Burns and Mitchell (1946) are, even after 60 years, still commonly used, even more so as their study refers to a period before the second world war (1885 to 1931). However, since the second world war many important characteristics of the classical business cycle have changed. The service sector for example increased heavily; other sectors, such as the agriculture sector, underwent a decline. In addition to these sectoral transformations economic developments, such as the emergence of new central banks or the unification of Europe, took place. Already Burns and Mitchell pointed out that the characteristics of business cycles are altering with the course of time: They showed that the average duration of business cycles was shorter in the period from 1915 to 1931 than in the period from 1885 to 1915, and thus, made clear that the minimum and maximum duration of business cycles may change. However, these results seem not to find their way into the current literature.

The following study attempts to define the minimum and maximum duration of business cycles since 1955 (thus, since the beginning of the postwar period). In the literature several methods are suggested for this purpose. In general they can be distinguished between non-parametric and parametric methods. To the category of non-parametric methods belongs the Bry and Boschan (1971) procedure, which requires no statistical model to determine the turning points of a data series. ${ }^{2}$ The second category, namely the parametric methods, can be subdivided into four classes. Authors like Beveridge and Nelson (1981), Nelson and Plosser (1982) and Campbell and Mankiw (1987) define the first class.

\footnotetext{
${ }^{2}$ Other non-parametric methods are those from Diebold and Rudebusch (1990), Diebold, Rudebusch, and Sichel (1991) and Watson (1994), however these methods require more than one data series.
} 
They consider ARMA or ARIMA processes in order to characterize business cycles. The second class, which is dominated by authors such as Harvey (1985), Watson (1986) or Clark (1987), utilizes linear unobserved components models for the analysis of the characteristics of business cycles. A third class of methods follows the co-integration specification of Engle and Granger (1987). These three approaches are all based on the assumption that the first difference of the log of the gross domestic product follows a linear stationary process. The fourth class embraces the methods of Hamilton (1989) and Filardo and Gordon (1998); they assume that the first difference of the log of the gross domestic product follows a non-linear stationary process, and apply Markov processes to define the characteristics of business cycles. ${ }^{3}$

If the goal is the utilization of a method as automatic as possible, making as few assumptions concerning the business cycle as possible, only the nonparametric procedure of Bry and Boschan (1971) is to be considered. Harding and Pagan (2003) compared the method by Hamilton (1989) with the one by Bry and Boschan (1971) and came to the conclusion that the Bry and Boschan procedure is more appropriate to reach the aforesaid goal. The Bry and Boschan procedure is relatively widespread and requires only very few assumptions to determine the turning points of a data series; the minimum and maximum duration can then be derived very easily based on these turning points. The following chapter describes the Bry and Boschan (1971) procedure and develops a computer algorithm thereto.

\section{The Bry and Boschan Procedure}

The algorithm by Bry and Boschan (1971, p. 21) is based upon the method of the National Bureau of Economic Research. In comparison to the method of the National Bureau of Economic Research, which is built upon several data

\footnotetext{
${ }^{3}$ Additional to the parametric and non-parametric methods there also exist hybrid methods, as for example those of Forni, Hallin, Lippi, and Reichlin (2000) and Artis, Krolzig, and Toro (2004).
} 
series, the algorithm by Bry and Boschan requires only one data series for the identification of the turning points. This simplifies the calculation, but also leads to certain imprecisions.

The Bry and Boschan (1971) procedure has already been applied several times in the literature and is regarded as a practical and straightforward method to compute the turning points of a time series (in the majority of cases the gross domestic product). Amongst others, the procedure was addoped by King and Plosser (1994), Watson (1994), Pedersen (1998) and Harding and Pagan (2002).

The procedure byBry and Boschan (1971) is comprehensibly structured. At first the time series to be analyzed is being highly smoothed in order to determine the approximate time of the turning points, or rather narrow down the region in which the turning points are likely to be located. Afterwards the smoothing is being reduced in various gradings until the original time series is at hand. After each reduction of the smoothing the turning points are being adjusted (however not newly determined anymore), so that in the end the exact period, in which the peaks and the troughs occur, results.

This procedure is particularly suited for data series with many local minima and maxima, as observed in most business cycle indicators. ${ }^{4}$ Such time series quite often feature multiple peaks or troughs which follow each other, and consequently no obvious cycle which could be assessed.

More precisely, the Bry and Boschan (1971) procedure consists of six steps. In a first step, outliers and extreme values are removed. The second step is about the smoothing of the time series with a moving average and the determination of the approximate turning points. Thereby it is imperative to pay attention to the circumstance that a peak always follows a trough and vice versa. In the third step, the smoothing of the time series is reduced and the turning points are adjusted to the new time series. Furthermore, a minimum duration for the

\footnotetext{
${ }^{4}$ Considered as business cycle indicator are amongst others: gross national product or gross domestic product, industrial production, unemployment rate, consumer prices, retail trade volume, export and import volume, money supply, commodity prices, private consumption, governmental expenditures, investments in equipment, investments in construction, changes in stock, exports (goods and services), and imports (goods and services).
} 
cycles is enforced. In the fourth step, the smoothing is reduced again and the turning points are readjusted. The fifth step readjusts the turning points in respect to the original time series and again enforces a minimum duration for the cycles (as in the third step). In the sixth step, the turning points which were calculated in the five previous steps are displayed.

Most probably the Bry and Boschan (1971) procedure is so popular because only very few assumption have to be made. Such assumptions were defined by Bry and Boschan for monthly data. However, as the data series for the gross domestic product is only available in the form of quarterly data, the assumptions have to be adjusted. Hence in the following the five assumptions which form the basis of the presently developed quarterly Bry and Boschan procedure are defined. The first three assumptions, which include the crucial points, were already mentioned in the preceding paragraph, however to report in full, they are defined once more and connected with a value.

Table 1: Assumptions for the quarterly Bry and Boschan procedure

1. A peak (trough) must be followed by a trough (peak).

2. A phase (from peak to trough or from trough to peak) must have a duration of at least 2 quarters.

3. A cycle (from peak to peak or from trough to trough) must have a duration of at least 5 quarters.

4. Turning points are not to be situated within the first or last 2 quarters of a time series.

5. The first (last) peak and trough must be higher respectively lower than values closer to the beginning (end) of the data series.

Most variations from the original Bry and Boschan procedure are relatively obvious. The translation from monthly periods into quarterly periods only causes a problem with the values of 4 and 5 months, as these would theoretically correspond to 1.333 and 1.667 quarters respectively. The procedure described here was tested with three different options of rounding: normal rounding, rounding up to the next integer, and rounding down to the next integer. The empirical tests in chapter 4 showed that strict normal rounding is best suited to reflect the data of the gross domestic product. In other words 4 months correspond to 1 quarter, 5 months correspond to 2 quarters, et cetera. 
However, the quarterly Bry and Boschan procedure developed in table 2 was not only adjusted to quarterly data but also slightly modified; the applied modifications are described in full detail in chapter $4 .^{5}$

Table 2: Modified Bry and Boschan procedure

1. Determination of extreme values and and substitution of these values.

2. Determination of cycles in a 2-quarter moving average (extreme values replaced) ${ }^{\dagger}$

a Identification of points higher (or lower) than 2 quarters on each side.

b Enforcement of alternation of turning points by selecting the highest of multiple peaks (or lowest of multiple troughs).

3. Determination of the corresponding turning points in the Spencer curve (extreme values replaced).

a Identification of the highest (or lowest) value within 2 quarters of the selected turning point in the 2-quarter moving average. ${ }^{\dagger}$

b Enforcement of a minimum cycle duration of 5 quarters by eliminating lower peaks and higher troughs of shorter cycles.

4. Determination of the corresponding turning points in the original data series or a 2quarter moving average, depending on the QCD (quarter of cyclical dominance).*

a Identification of the highest (or lowest) value within 2 quarters of the selected turning points in the Spencer curve.

5. Determination of the turning points in the original data series.

a Identification of the highest (or lowest) value within the QCD term of the selected turning points in the 2-quarter moving average or the original data series.*

b Elimination of turning points within 2 quarters of the beginning and the end of the data series.

c Elimination of peaks (or troughs) at both ends of the data series which are higher (or lower) than values closer to the end.

d Elimination of cycles whose duration is less than 5 quarters.

e Elimination of phases whose duration is less than 2 quarters.

6. Statement of the final turning points.

$\dagger$ : To be exactly accurate a 4-quarter moving average should be used. But chapter 4 shows that a 2-quarter moving average better reflects the reality.

* : The QCD values are per definition either 1 or 2 .

For the programming in MatLab the monthly Bry and Boschan procedure from Watson (1994), which was written in Gauss, was adapted and partially modified. Three authors are known to also make use of the Bry and Boschan procedure for quarterly data: Pedersen (1998), Harding and Pagan (2002), and Inklaar, Jacobs, and Romp (2003). However, these procedures are either not available or cannot resolve all doubt. ${ }^{6}$ For example Inklaar, Jacobs, and Romp

\footnotetext{
${ }^{5}$ The procedure was programmed in MatLab and is available upon request.

${ }^{6}$ The doubts concerning the procedure from Harding and Pagan (2002) are expressed in
} 
overlooked a mistake while programming their procedure. They are using a 15-point Spencer curve for quarterly data, which in practice is relatively often used to smooth monthly data. Kendall and Stuart (1966) show that a 15point Spencer curve features approximately the same reduction as a moving average of 5. It is therefore apparent that the smoothing characteristics of the Spencer curve have to be adjusted when using quarterly data. Therefore a 5 -point Spencer curve with the weights

$$
\frac{1}{35}[-3,12,17,12,-3]
$$

is used in the following. The same Spencer curve is also used to identify the outliers and extreme values of the data series.

\section{Verification of the Bry and Boschan procedure}

To verify the validity of the just developed quarterly Bry and Boschan procedure the turning points found with the procedure are in the following compared to the official data on the characteristics of business cycles.

The United States of America are the only country where a committee (the National Bureau of Economic Research) exists which officially announces data on the turning points of business cycles. Hence in the following the turning points of the gross domestic product of the United States of America are calculated by means of the quarterly Bry and Boschan procedure. These turning points are then compared with the official data from the National Bureau of Economic Research. The turning points are calculated and analyzed for the period between 1955 and 2004 .

Since 1955 the National Bureau of Economic Research registered 7 business cycles with a minimum duration of 18 months or 6 quarters and a maximum duration of 128 months or 43 quarters. As already mentioned in chapter 2, Burns and Mitchell (1946) defined the minimum duration to 16 to 22 months

footnote 11 . 
and the maximum duration to 100 to 106 months, respectively 5 to 7 and 33 to 35 quarters. In other words: The minimum duration did not change since the analysis conducted by Burns and Mitchell, the maximum duration of a business cycle however seems to have been prolonged by approximately 2 years over the same period.

Therefore, to measure the minimum and maximum duration of business cycles in the United States, the Bry and Boschan procedure is basically not necessary, but just a quick analysis of the data from the National Bureau of Economic Research will do. ${ }^{7}$ Facing this fact it is not precisely pursuable why known authors such as King and Plosser (1994), Watson (1994) or Baxter and King (1999) still use the data published by Burns and Mitchell (1946) for their calculations instead of accessing the current data of the National Bureau of Economic Research.

As the characteristics of the business cycles of the United States of America are announced by the National Bureau of Economic Research and as these data are often used as benchmark in the literature, the quarterly procedure developed in chapter 3 will in the following be evaluated and examined by means of this data. Additionally, the two modifications of the procedure will be analyzed.

In comparing the turning points of the gross domestic product of the United States calculated with the unmodified quarterly Bry and Boschan procedure and the official data published by the National Bureau of Economic Research, it becomes evident that the Bry and Boschan procedure is too restrictive. The unmodified procedure only finds 4 business cycles, while the National Bureau of Economic Research defines 7 cycles. A deeper analysis of the procedure shows that mainly step 2 of the unmodified quarterly Bry and Boschan procedure, which is about the determination of the cycles in a smoothed data series, is too restrictive.

The restrictions in step 2 (the individual steps are listed in table 2) of the

\footnotetext{
${ }^{7}$ As the National Bureau of Economic Research understandingly only defines the business cycles for the United States of America, the quarterly Bry and Boschan procedure remains acutely useful for the determination of the business cycles of all other countries.
} 
quarterly Bry and Boschan procedure could be released in two possible ways. Either step 2a, namely the identification of points higher (or lower) than 5 months on either side, could be loosened in rounding down the original indication of 5 months and translating it with 1 quarter, or step 2 , in other words the determination of the cycles in a 12-months moving average, could be made less tight in not determining the cycles in a 4-quarter but a 2-quarter moving average.

Table 3 compares the data from the National Bureau of Economic Research (NBER) with three quarterly Bry and Boschan procedures: A modified Bry and Boschan procedure with a 2-quarter moving average (MBBP step 2), a modified Bry and Boschan procedure with rounding down the 5 months indication (MBBP step 2a), and a unmodified Bry and Boschan procedure (QBBP). ${ }^{8}$

Table 3: Duration of business cycles and phases since 1955

\begin{tabular}{|c|c|c|c|c|c|c|}
\hline & \multirow{2}{*}{$\begin{array}{l}\text { Number } \\
\text { of cycles }\end{array}$} & \multicolumn{3}{|c|}{ Business cycle duration } & \multicolumn{2}{|c|}{ Mean duration of } \\
\hline & & $\min$ & $\max$ & mean & recession & boom \\
\hline QBBP & 4 & 6 & 68 & 33 & 2.6 & 30.25 \\
\hline MBBP (step 2a) & 6 & 6 & 57 & 28.92 & 2.71 & 26.17 \\
\hline MBBP (step 2) & 7 & 6 & 42 & 24.79 & 2.63 & 22.14 \\
\hline NBER & 7 & 6 & 43 & 24.86 & 3.25 & 21.57 \\
\hline
\end{tabular}

It becomes evident in table 3 that, the results change relatively strongly with the first modification (step 2a), namely the identification of points higher (or lower) than only 1 quarter on either side. Two of the three business cycles which are missing in the unmodified procedure are now included in the procedure. ${ }^{9}$ Indeed, the maximum duration fits the benchmark data clearly better, while with 57 quarters it is still relatively far away from the 43 quarters determined by the National Bureau of Economic Research.

With the second modification (step 2), which is the determination of the cycles in a 2-quarter moving average, the results again improve unambiguously. With this modification all business cycles are contained in the procedure $;^{10}$ in

\footnotetext{
${ }^{8}$ Table 2 shows the modified Bry and Boschan procedure with a 2-quarter moving average.

${ }^{9}$ These are the business cycles from Quarter II 1960 to Quarter I 1961 and Quarter I 2001 to Quarter IV 2001.

${ }^{10}$ Also the cycle from Quarter IV 1969 to Quarter IV 1970 is now included.
} 
addition it very precisely maps the maximum duration of the business cycles onto the duration defined by the National Bureau of Economic Research with a difference only one quarter.

As a combination of both modifications does not lead to additional accuracy, only the modification of step 2, which uses a 2-quarter moving average instead of a 4-quarter moving average to determine the cycles, will be applied in the following. The procedure to this modification is described in detail in chapter 3 , table $2 .^{11}$

In addition table 3 shows that an average recession phase lasts approximately 3 quarters, boom phases on the other hand last 22 quarters. Hence booms last on average 18 to 19 quarters longer than recessions.

Of further interest is also the confirmation that the maximum duration of business cycles in the United States of America has clearly been extended since Burns and Mitchell (1946). If authors like King and Plosser (1994), Stock and Watson (1998), or Baxter and King (1999) still assume a maximum duration of 32 quarters, they distinctly underestimate it. They should also adopt the actual maximum duration of 42 to 43 quarters.

Figure 1: Comparison of the turning points by NBER and MBBP - NBER, MBBP

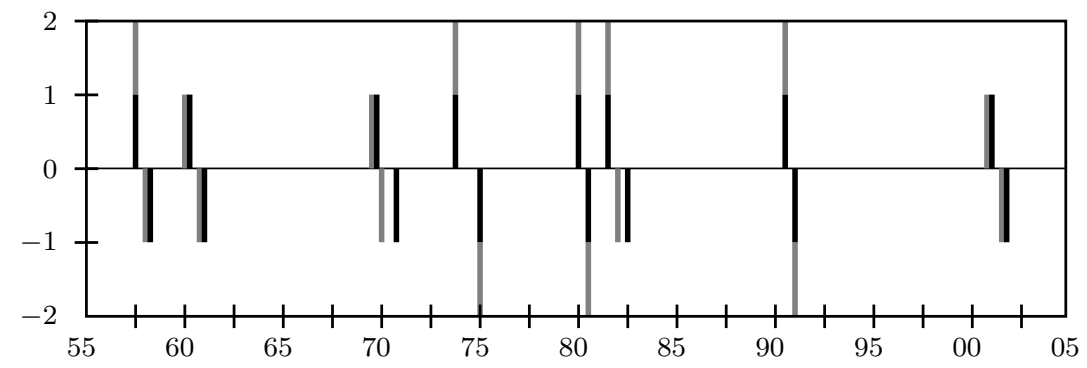

By means of table 3 the precision of the procedure developed in chapter 3 regarding the minimum and maximum duration was examined. But from this table it is not evident whether the modified Bry and Boschan procedure deter-

\footnotetext{
${ }^{11}$ Although Harding and Pagan (2002) did not mention any deviations from the original procedure, it has to be assumed that they also did not filter with a 4-quarter moving average but used some modification. The peak in Quarter IV 1969 as well as the trough in Quarter IV 1970 are only apparent with a 2 -quarter or 3-quarter moving average.
} 
mines the appropriate time of the turning points. Figure 1 shows the turning points defined by the National Bureau of Economic Research (NBER) in comparison to the turning points calculated with the modified Bry and Boschan procedure (MBBP).

Figure 1 proves that the modified Bry and Boschan procedure reproduces the data from the National Bureau of Economic Research very well. As the turning points determined by the National Bureau of Economic Research are based on monthly data, it is possible that differences of one quarter arise. Discrepancies of more than 1 quarter only occur twice, namely at the trough of Quarter IV 1970 and at the trough of Quarter IV 1982. ${ }^{12}$

The discrepancy of Quarter IV 1970 could be removed with a modification of step 5a of the quarterly Bry and Boschan procedure, so that the lowest values are not identified within 1 quarter but within 3 quarters. However, as this represents a rather large intervention to the original procedure, this modification will not be undertaken.

The second discrepancy, which is the one of Quarter IV 1982, cannot be resolved, as the data series of the gross domestic product reaches the trough in Quarter I 1982. From the gross domestic product data a second trough appears in Quarter III 1982; but this trough lies higher than the trough of Quarter I 1982.

In conclusion, it can be said that the Bry and Boschan procedure developed in chapter 3 adequately reproduces the turning points published by the National Bureau of Economic Research. The duration of the boom and recession phases, the duration of the business cycles, as well as the time of the turning points fit in astoundingly well.

After having successfully tested the quarterly Bry and Boschan procedure in this chapter, the next chapters apply the procedure to sectorial and industrial data of the United Kingdom.

\footnotetext{
${ }^{12}$ Both troughs appear in the quarterly Bry and Boschan procedure three quarters too early.
} 


\section{$5 \quad$ Data and Definitions}

The United Nations (2003) publish data of the gross domestic product for 179 countries and regions, among them also the gross value added for 16 industries in constant prices.

Unfortunately these data are only published yearly by the United Nations. However, similar data are available from the Office for National Statistics of the United Kingdom: The Office for National Statistics publishes quarterly indices at constant prices of the gross value added for 16 industries, which are also analyzed in the United Nations statistics. In the following these 16 industries are, as shown in table 4 , summarized in 4 sectors and 14 industries. ${ }^{13}$

Table 4: Classification of sectors and industries

1. Agriculture, hunting, forestry and fishing

2. Production

2.1. Mining and quarrying

2.2. Manufacturing

2.3. Electricity, gas and water supply

3. Construction

4. Service

4.1. Wholesale, retail trade, repair of motor vehicles, motorcycles and personal and household goods

4.2. Hotels and restaurants

4.3. Transport, storage and communication

4.4. Financial intermediation

4.5. Real estate, renting and business activities

4.6. Public administration and defence, compulsory social security

4.7. Education

4.8. Health and social work

4.9. Other community, social and personal services

The data on the gross domestic product belonging to these statistical series is only available since 1955. Therefore series available for a longer period of time will in the following only be analyzed since 1955. As most European economies, and certainly also the one of the United Kingdom, were strongly affected by

\footnotetext{
${ }^{13}$ Whereas the agriculture and construction sector are also counted as industries.
} 
the war and post-war events until the fifties, this circumstance should not be valued too negatively. It can be assumed that most economies recovered from these events until the year of 1955, and hence, that since 1955 an unaltered gross domestic product is at hand.

In the following, the quarterly Bry and Boschan procedure developed in chapter 3 is calculated for the sectors and industries classified in table 4 to determine the duration of the business cycles of the individual sectors and industries respectively. For reasons of simplicity the agriculture and construction sector, which cannot be segmented into different industries, are thereby treated as a sector as well as an industry.

\section{Duration of the business cycles by sector}

As mentioned in chapter 2 a standardized procedure is needed for calculating the duration of the business cycles of the gross value added of sectors and industries.

Table 5 shows the duration of sectorial business cycles, calculated with the modified Bry and Boschan procedure developed in chapter 3. The breakdown of the overall economy into different sectors corresponds to the classification described in table 4. Each business cycle lasts from peak to peak or trough to trough. If the number of peak to peak cycles differs from the number of trough to trough cycles, the higher value is selected.

Table 5: Duration of sectorial business cycles and phases since 1955

\begin{tabular}{lc|ccc|cc}
\hline \hline & $\begin{array}{c}\text { Number } \\
\text { of cycles }\end{array}$ & \multicolumn{2}{c}{ Business cycle duration } & \multicolumn{2}{c}{ Mean duration of } \\
& 13 & 6 & 30 & 14.8 & 4.75 & 9.92 \\
\hline Agriculture & 13 & 7 & 32 & 16.68 & 4.17 & 12.72 \\
Production & 11 & 7 & 29 & 17.35 & 6.18 & 10.9 \\
Construction & 10 & 41 & 100 & 61.33 & 6 & 65.5 \\
Services & 2 & $\mathbf{4}$ & $\mathbf{4 9}$ & $\mathbf{2 3 . 2 5}$ & $\mathbf{4 . 2 9}$ & $\mathbf{1 9}$ \\
GDP & $\mathbf{6}$ & $\mathbf{5}$ & & & &
\end{tabular}

It is evident from table 5 that since 1955 the United Kingdom had 6 business cycles, with a mean duration of 23.25 quarters. The shortest business cycle in the United Kingdom lasted 5 quarters, the longest 49 quarters. 
Burns and Mitchell (1946, p. 441) estimated the duration of business cycles in Great Britain on minimal 16 to 22 months and maximal 135 to 141 months, whereby they used data from 1854 to 1932 for their calculations. In quarters, this would correspond to a minimum duration of 5 to 7 quarters and a maximum duration of 45 to 47 quarters. Contrary to chapter 4 for the United States of America, it cannot be shown for the United Kingdom that the maximum duration of business cycles prolonged since 1932, as the difference to the results of Burns and Mitchell (1946) only accounts for 2 quarters. Yet, unfortunately many authors did not use the correct data from Burns and Mitchell (1946) for the United Kingdom, but the 6 and 32 quarters recommended for the United States of America by Baxter and King (1999). ${ }^{14}$ However, filters, such as those from Baxter and King (1999) or Christiano and Fitzgerald (2003) should be adjusted. In case these filters are calculated with the values proposed by the authors ( 6 and 32 quarters), business cycles with a duration between 32 and 49 quarters would disappear.

If the data compiled for the gross domestic product of the United Kingdom in table 5 is compared with the data for the United States of America in table 3, certain common characteristics attract attention. The minimum and maximum duration of the business cycles show a great resemblance for both countries. Also the mean duration of business cycles is only approximately one and a half quarters shorter in the United Kingdom than in the United States of America. Upon direct comparison of the data of the turning points, it becomes apparent that the timing of the business cycles of both countries is astonishingly similar, too. Only the recessions in the United States of America of 1969/70 and 2001 did not appear in the United Kingdom. In return, the United Kingdom had a recession in 1955/65 which did not occur in the United States of America.

Likewise apparent in table 5 is that in the United Kingdom the boom phases lasted on average 14.71 quarters longer than the recession phases. This is not

\footnotetext{
${ }^{14}$ For example Bergman (2004) as well as Bergman, Bordo, and Jonung (1998) refer in their country study on the specification from Baxter and King (1999) which was explicitly calculated for the United States of America.
} 
only the case for the overall economy (which is being represented by the gross domestic product), but also for the individual sectors. Booms in the different sectors last on average even 19.49 quarters longer than recessions. The difference between boom and recessions phases is, with 4.72 quarters, smallest in the construction sector and, with 59.5 quarters, largest in the service sector. However it must be emphasized that the service sector only registered two business cycles since 1955; hence the boom phases in this sector are, with 65.5 quarters on average, extraordinarily large.

As no detailed trend analysis was conducted, and as the analysis in table 5 merely relates to the duration of cycles and phases of the gross value added of individual sectors and not to their long-term growth, no qualitative conclusions about the growth of the sectors can currently be drawn.

\section{Duration of the business cycles by industry}

After the business cycles and phases of the 4 sectors of the United Kingdom took center stage in chapter 6 , this chapter will analyze the business cycles and phases of the 14 industries from table 4 .

Unfortunately, the gross value added for the individual industries is not always available since 1955. Therefore, it is not directly evident from table 6 which industries exhibit the most business cycles. By means of the lengths of the data series and the number of business cycles, a 10-year average amount of business cycles of an industry can be calculated. The mining and quarrying as well as the agriculture industry were, with on average 2.6 business cycles, most affected by cyclical fluctuations, followed by the manufacturing industry with 2.4 and the industries electricity as well as public administration each with 2.2 business cycles on a 10-year average.

On the other side of the spectrum are the wholesale and retail trade industry with only 0.94 business cycles, the transport, storage and communication industry with 1 , and also the industries financial intermediation, real estate and 
other services each with 1.05 cycles on a 10-year average.

It is not surprising that industries such as manufacturing or electricity dispose more business cycles than industries such as whole and retail trade or financial intermediation, as it is common knowledge that the first category of industries is more exposed to cyclical fluctuations than the latter.

Once more it must be emphasized, that the amount of business cycles does not reveal the growth of an industry. On the other hand, a correlation between cyclical industries and the amount of business cycles can be observed. High amplitudes are expected from cyclical industries, but not necessarily a high frequency. However, table 6 shows that the cyclical industries manufacturing, electricity, mining and quarrying, and hotels and restaurants, have relatively many business cycles, that is a high frequency. The less cyclical industries transport, storage and communication, wholesale and retail trade, and financial intermediation in contrary exhibit relatively few business cycles, that is a low frequency.

Table 6: Duration of industrial business cycles and phases since 1955

\begin{tabular}{|c|c|c|c|c|c|c|}
\hline & \multirow{2}{*}{$\begin{array}{l}\text { Number } \\
\text { of cycles }\end{array}$} & \multicolumn{3}{|c|}{ Business cycle duration } & \multicolumn{2}{|c|}{ Mean duration of } \\
\hline & & $\min$ & $\max$ & mean & recession & boom \\
\hline Agriculture & 13 & 6 & 30 & 14.8 & 4.75 & 9.92 \\
\hline Mining & 13 & 5 & 39 & 13.76 & 8.23 & 6 \\
\hline Manufacturing & 12 & 6 & 31 & 15.21 & 4.38 & 10.92 \\
\hline Electricity & 11 & 6 & 44 & 15.59 & 4 & 12.18 \\
\hline Construction & 10 & 7 & 29 & 17.35 & 6.18 & 10.9 \\
\hline Wholesale, Retail $^{\dagger}$ & 3 & 6 & 43 & 23 & 4 & 16.33 \\
\hline Hotels* & 7 & 5 & 41 & 18.85 & 6 & 12.57 \\
\hline Transport & 5 & 7 & 57 & 27.44 & 4.5 & 21.8 \\
\hline Financial $^{* *}$ & 2 & 6 & 32 & 20.25 & 3.67 & 17 \\
\hline Real Estate** & 2 & 6 & 35 & 20 & 4.67 & 14.5 \\
\hline Public Admin. & 11 & 5 & 23 & 14.43 & 7.6 & 7 \\
\hline Education $^{\dagger}$ & 6 & 10 & 26 & 18.09 & 3.83 & 14.8 \\
\hline Health $^{\dagger}$ & 0 & - & - & - & - & - \\
\hline Other Services** & 2 & 6 & 49 & 27 & 3.67 & 23.5 \\
\hline GDP & 6 & 5 & 49 & 23.25 & 4.29 & 19 \\
\hline
\end{tabular}

When analyzing the average duration of boom- and recession phases caution should be exercised. Chapter 4 showed that the maximum duration of business cycles in the United States of America increased since 1932. As not all industries have the same lengths of data series, a comparison between the average duration 
of different industries is not unproblematic. However, as the differences in the length of the data series of many industries are not too big, a quick analysis of the cycles and phases shall be attempted in the following.

Table 6 shows that the industries mining and quarrying, public administration and construction dispose notedly long boom- and recession phases; in the mining and quarrying and the public administration industry recessions on average even last longer than booms. For all other industries the same result applies as for the sectors in chapter 6: boom phases normally last longer than recession phases. On average for all industries booms last 8.61 quarters (hence more than 2 years) longer than recessions. As already mentioned this indicator is being falsified trough the circumstance that some industries dispose a shorter data set. The indicator for the gross domestic product is more accurate: As already shown in the analysis concerning the sectors in chapter 6 , the boom phases of the gross domestic product last on average 14.71 quarters longer than the recession phases.

\section{Implications for band-pass filters}

Chapters 4, 6 and 7 showed that the maximum duration of the business cycles of the gross domestic product strongly differs from the value proposed by Baxter and King (1999), Christiano and Fitzgerald (2003) and others. Business cycles in the United States of America last for a minimum of 6 and a maximum of 42 quarters, while in the United Kingdom they last for a minimum of 5 and a maximum of 49 quarters.

Furthermore, it has been illustrated in chapter 6 and 7 , that the minimum and maximum duration of the business cycles sometimes strongly differ among individual sectors and industries. Table 6 for instance shows that the maximum duration varies, depending on the industry, between 23 and 57 quarters.

The differences to the minimum and maximum durations of Burns and Mitchell (1946) as well as the large differences of the durations of the inid- 
vidual sectors and industries elucidate, that in order to correctly apply filter techniques such as the Baxter and King (1999) or the Christiano and Fitzgerald (2003) filter, it is essential to know the minimum and maximum duration of the cycles under investigation.

The filter by Baxter and King as well as the one by Christiano and Fitzgerald divide data series into a long-term trend, a medium-term business cycle and short-term noise. All cycles which are shorter than the minimum duration are regarded as short-term noise, those longer than the maximum duration as longterm trend. Hence, if for instance the gross domestic product of the United States of America is filtered with a maximum duration of 32 quarters (instead of 42 quarters), as it was proposed by Baxter and King (1999), the cycles with a duration between 32 and 42 quarters are interpreted as long-term trend and not as medium-term business cycles. Consequently, the amplitudes of the mediumterm business cycles are underestimated, while the variability of the growth rate of the long-term trend is overestimated. ${ }^{15}$

As described in Everts (2006), longer business cycles are better reproduced with the filter from Christiano and Fitzgerald (2003) than with the one from Baxter and King (1999).

Therefore, in the following the Christiano and Fitzgerald filter with the minimum and maximum duration of 6 and 32 quarters, as proposed by the authors, is compared to a filter with the real minimum and maximum duration, as determined in the chapters 4,6 and $7 .^{16}$ Afterwards the differences of the two calculation methods on the medium-term business cycles and the long-term trend is analyzed. In a first step this proceeding is applied to the gross domestic product of the United States of America, in a second step to the gross domestic product of the United Kingdom, and in a third step to the individual sectors and industries of the United Kingdom.

Figure 2 shows the medium-term business cycles and the long-term trend of

\footnotetext{
${ }^{15}$ It requires no interpretation that the variability of the medium-term business cycles increases, if the maximum duration is set higher, and hence more business cycles are included.

${ }^{16}$ The filter was programmed in MatLab and is available upon request.
} 
the gross domestic product of the United States of America. The business cycle and the trend are once calculated with a minimum and maximum duration of 6 and 32 quarters, and once with a minimum and maximum duration of 6 and 42 quarters. $^{17}$

Figure 2: Business cycles and trend in the United States of America - 632 quarters, 6 \& 42 quarters
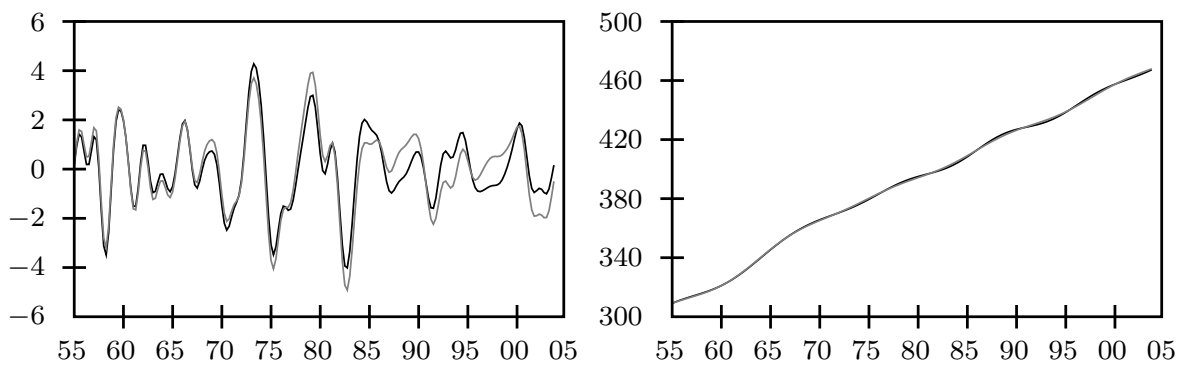

From figure 2 it appears that the business cycles filtered with a maximum duration of 42 quarters display a higher amplitude than the business cycles filtered with a maximum duration of just 32 quarters. To estimate the difference of the amplitudes, the average amplitude at the turning points determined with the procedure developed in chapter 3 is being calculated. The average amplitude of the turning points is $23 \%$ higher when using a filter with a maximum duration of 42 quarters instead of a filter with a maximum duration of 32 quarters. ${ }^{18}$

Figure 2 also reveals that the growth rate of the long-term trend with a minimum duration of 42 quarters varies less than the growth rate of the trend with a minimum duration of 32 quarters. If a filter with a maximum duration of 32 quarters is applied, the growth rate of the long-term trend in the period between 1979 and 1983 apparently is underestimated, between 1983 and 1988 overestimated, and subsequently - in the period from 1988 to 1993 - again underestimated. To determine this difference in the variability, the standard deviation of the growth rate of the long-term trend is being calculated. For

\footnotetext{
${ }^{17}$ All filters are calculated on the logarithm of the gross domestic product or the gross value added respectively. For the diagrams the values are multiplied with 100 in order to emphasize differences in size.

${ }^{18}$ If the average amplitudes are calculated at the turning points determined by the National Bureau of Economic Research, the average amplitude for the filter with a maximum duration of 42 quarters is $9.36 \%$ higher than for the filter with a maximum duration of 32 quarters.
} 
the filter with a maximum duration of 42 quarters the standard deviation is $0.07 \%$, for the filter with a maximum duration of 32 quarters $0.08 \%$. Hence the variability of the long-term trend would be underestimated by $11.69 \%$, if a filter with a maximum duration of 32 instead of 42 quarters is applied.

The two effects become even more apparent, if - in a second step - the gross domestic product of the United Kingdom is analyzed instead of the of the United States of America. As pointed out in chapter 6, some authors refer to the studies of Baxter and King (1999) and therewith indirectly to Burns and Mitchell (1946) also for other countries than the United States of America, even tough Burns and Mitchell determined the duration of the business cycles for Great Britain, France and Germany likewise.

Table 5 makes evident that the minimum duration of the business cycles in the United Kingdom is 5 quarters and the maximum duration 49 quarters. Hence figure 3 compares a Christiano and Fitzgerald (2003) filter with minimum and maximum durations of 6 and 32 quarters, as spuriously being used in practice, with a filter with minimum and maximum duration of 5 and 49 quarters, as it should be deployed correctly.

Figure 3: Business cycles and trend in the United Kingdom

- 6 \& 32 quarters, 5 \& 49 quarters
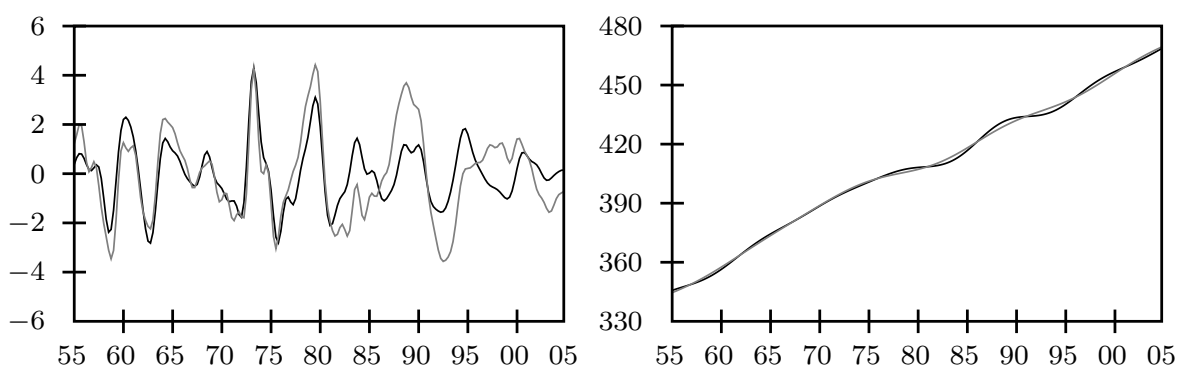

Figure 3 shows, that for the United Kingdom the amplitudes of the business cycle are considerably higher for the filter with a maximum duration of 49 quarters than for the filter with a maximum duration of 32 quarters. The average amplitude at the turning points is $40.02 \%$ higher for the filter with a maximum duration of 49 quarters than for the filter with a maximum duration 
of 32 quarters.

It is remarkable that the filters partly display relatively different business cycles for the United Kingdom. When looking at the long-term trend, it becomes evident why the business cycles proceed so differently: The filter with a maximum duration of 32 quarters heavily underestimates the growth rate of the long-term trend between 1978 and 1983, overestimates it between 1983 and 1988, again underestimates it between 1989 and 1992. Once more the standard deviation of the growth rate of the long-term trend is calculated. For the filter with a maximum duration of 49 quarters it amounts to $0.04 \%$, for the filter with a maximum duration of 32 quarters to $0.07 \%$. Hence the variability of the long-term trend of the United Kingdom is by $32.29 \%$ smaller for a filter with a maximum duration of 49 quarters.

In conclusion it can be said that the variability of the growth rate of the longterm trend is being overestimated, and that the amplitudes of the medium-term business cycles are being underestimated, if the maximum duration is chosen to small. If the maximum duration is too big, the variability of the growth rate of the long-term trend is being underestimated, and the amplitudes of the medium-term business cycles overestimated.

Similar results hold for the over- and underestimation of the minimum duration. If the minimum duration is chosen too small, the amplitudes of the medium-term business cycles are overestimated. But if the minimum duration is chosen too big, the amplitudes of the medium-term business cycles are underestimated.

In a next step, the over- and underestimation of the average amplitudes of the medium-term business cycles as well as of the variability of the growth rate of the long-term trend are being calculated in table 7 and 8 . The data each refer to the difference between a filter with a minimum duration of 6 quarters and a maximum duration of 32 quarters and a filter with the real minimum and maximum duration. The real minimum and maximum duration, which was calculated in chapter 6 and 7 , are once again reproduced. 
Table 7: Over- and underestimation of the sectoral business cycles and trends since 1955

\begin{tabular}{lcccc}
\hline \hline & \multicolumn{2}{c}{ Cycle Duration } & Amplitude & Trend \\
& Min & Max & & \\
\hline Agriculture & 6 & 30 & $-0.37 \%$ & $1.01 \%$ \\
Production & 7 & 32 & $-6.88 \%$ & $0.00 \%$ \\
Construction & 7 & 29 & $-2.51 \%$ & $5.36 \%$ \\
Services & 41 & 100 & $-44.35 \%$ & $-63.94 \%$ \\
GDP & $\mathbf{5}$ & $\mathbf{4 9}$ & $\mathbf{4 0 . 0 2 \%}$ & $\mathbf{- 3 3 . 2 9 \%}$ \\
\hline
\end{tabular}

Some of the data for the sectors of the United Kingdom in table 7 are unfortunately not unambiguously interpretable. The production sector however shows clearly that the average amplitudes of business cycles are smaller if the filter is calculated with a minimum duration of 7 instead of 6 quarters. Furthermore, the construction sector and the service sector demonstrate that the variability of the growth rate of the long-term trends increases (decreases), if the calculation is done with a shorter (longer) maximum duration.

Table 8: Over- and underestimation of the industrial business cycles and trends since 1955

\begin{tabular}{|c|c|c|c|c|}
\hline & \multicolumn{2}{|c|}{ Cycle Duration } & \multirow{2}{*}{ Amplitude } & \multirow{2}{*}{ Trend } \\
\hline & Min & $\operatorname{Max}$ & & \\
\hline Agriculture & 6 & 30 & $-0.37 \%$ & $1.01 \%$ \\
\hline Mining & 5 & 39 & $13.58 \%$ & $-1.54 \%$ \\
\hline Manufacturing & 6 & 31 & $-0.15 \%$ & $0.21 \%$ \\
\hline Electricity & 6 & 44 & $2.51 \%$ & $-5.94 \%$ \\
\hline Construction & 7 & 29 & $-2.51 \%$ & $5.36 \%$ \\
\hline Wholesale, Retail $^{\dagger}$ & 6 & 43 & $24.88 \%$ & $-16.76 \%$ \\
\hline Hotels* & 5 & 41 & $34.88 \%$ & $-30.12 \%$ \\
\hline Transport & 7 & 57 & $13.69 \%$ & $-37.54 \%$ \\
\hline Financial** & 6 & 32 & $0.00 \%$ & $0.00 \%$ \\
\hline Real Estate** & 6 & 35 & $11.97 \%$ & $-8.33 \%$ \\
\hline Public Admin. & 5 & 23 & $-5.71 \%$ & $6.69 \%$ \\
\hline Education $^{\dagger}$ & 10 & 26 & $-25.76 \%$ & $9.97 \%$ \\
\hline Health $^{\dagger}$ & - & - & - & - \\
\hline Other Services** & 6 & 49 & $24.13 \%$ & $-50.54 \%$ \\
\hline GDP & $\mathbf{5}$ & 49 & $40.02 \%$ & $-33.29 \%$ \\
\hline
\end{tabular}

Table 8 shows the over- respectively underestimation of the average amplitudes of the business cycles and of the variability of the growth rate of the trend for the individual industries of the United Kingdom. Table 8 shows that the average amplitudes of the medium-term business cycles are underestimated and the variability of the long-term trends overestimated, if the real maximum 
duration is clearly larger than 32 quarters, as for example in the industries mining and quarrying, electricity, wholesale and retail, hotels, transport, real estate, and other services. If the maximum duration is clearly smaller than 32 quarters, as in the industries public administration and education, the average amplitudes of the medium-term business cycles are overestimated and the variability of the growth rate of the long-term trend underestimated. However, in the education industry it is not unambiguously evident whether the overestimation of the amplitudes of the medium-term business cycles results from the shorter maximum duration or from the longer minimum duration.

Generally, the following can be stated: For an exact determination of the business cycles as well as the trends it is of utmost importance to know the minimum and maximum duration of the business cycles. Thus it is advised against applying the values of 6 and 32 quarters proposed by Baxter and King (1999) and Christiano and Fitzgerald (2003) without reflection. If the minimum duration is chosen too high (low), the amplitudes of the medium-term business cycles are overestimated (underestimated). And if one chooses the maximum duration to low (high), the average amplitudes of the business cycle are underestimated (overestimated) and the variability of the growth rate of the long-term trend is overestimated (underestimated).

\section{Conclusions}

If band-pass filters as those from Baxter and King (1999) or Christiano and Fitzgerald (2003) are employed, the question arises, what data to use for the minimum and maximum duration of the business cycles. For the the minimum and maximum duration the authors of the filters refer to the seminal paper of Burns and Mitchell (1946) but thereby neglect, that Burns and Mitchell relate in their calculations on a period between 1885 and 1931.

The article at hand consequently proposes a procedure which calculates the minimum and maximum duration of the business cycles by means of a single 
data series such as the gross domestic product or the gross value added. The proposed procedure is an amended Bry and Boschan (1971) procedure, which is modified to the extend that it is applicable to quarterly data such as the gross domestic product.

A comparison of the results of the proposed procedure with the specification of the National Bureau of Economic Research shows that the modified Bry and Boschan (1971) procedure developed in this study reproduces the business cycles of the United States of America very accurately. Furthermore, it can be shown that the maximum duration of the business cycles of the United States of America amounts to 42 quarters. Hence the maximum duration of business cycles clearly lies above the 32 quarters applied by Baxter and King (1999) and Christiano and Fitzgerald (2003) or proposed by Burns and Mitchell (1946).

A similar result arises for the United Kingdom: There, the maximum duration of the business cycles of the gross domestic product even amounts to 49 quarters. If additionally the minimum and maximum duration of the business cycles of the individual sectors and industries are compared by using the gross value added it becomes apparent that the minimum and maximum duration varies depending on the sector or industry.

Both outcomes, the finding that the maximum duration of the business cycles of the gross domestic product is clearly longer than assumed so far, and the outcome that the minimum and maximum duration differ by sector and industry, lead to the suspection that caution is advisable with the application of the filters by Baxter and King (1999) and Christiano and Fitzgerald (2003).

If the maximum duration for band-pass filters is chosen to low (high), the variability of the growth rate of the long-term trend is overestimated (underestimated) and the amplitudes of the medium-term business cycles are underestimated (overestimated). In other words: If the maximum duration for the filters by Baxter and King (1999) or Christiano and Fitzgerald (2003) is chosen too low, certain cyclical fluctuations are mistakenly interpreted as long-term trend and not as medium-term business cycles. On the other hand, if the maximum 
duration is chosen too high, some changes in trend are mistakenly interpreted as medium-term business cycles instead of long-term trend.

The study at hand shows the importance of the considerations that have to be taken into account when it comes to assessing which components of a data series belong to the medium-term cycles and which to the long-term trend; or in other words which maximum duration or frequency is considered to be the boundary between business cycles and changes in growth.

\section{References}

Artis, M. J., H.-M. Krolzig, And J. Toro (2004): "The European business cycle," Oxford Economic Papers, 56(1), 1-44.

Baxter, M., and R. G. King (1999): "Measuring Business Cycles: Approximate Band-Pass Filters For Economic Time Series," The Review of Economics and Statistics, 81(4), 575-593.

Bergman, M. (2004): "How Similar Are European Business Cycles?," Discussion Paper 2004:9, Lund University, Department of Economics.

Bergman, M., M. Bordo, and L. Jonung (1998): "Historical Evidence on Business Cycles: The International Experience," in Beyond Shocks: What Causes Business Cycles?, ed. by J. Fuhrer, and S. Schuh, no. 42 in Conference Series. Federal Reserve Bank of Boston.

Beveridge, S., and C. R. Nelson (1981): "A New Approach to Decomposition of Economic Time Series into Permanent and Transitory Components with Particular Attention to Measurement of the 'Business Cycle'," Journal of Monetary Economics, 7(2), 151-74.

Bry, G., And C. Boschan (1971): Cyclical Analysis of Time Series: Selected Procedures and Computer Programs. National Bureau of Economic Research, New York. 
Burns, A. F., And W. C. Mitchell (1946): Measuring Business Cycles. National Bureau of Economic Research, New York.

Campbell, J. Y., and N. G. Mankiw (1987): "Are Output Fluctuations Transitory?," The Quarterly Journal of Economics, 102(4), 857-80.

Christiano, L. J., and T. J. Fitzgerald (2003): "The Band Pass Filter," International Economic Review, 44(2), 435-65.

Clark, P. K. (1987): "The Cyclical Component of U.S. Economic Activity," The Quarterly Journal of Economics, 102(4), 797-814.

Diebold, F. X., And G. D. Rudebusch (1990): "A Nonparametric Investigation of Duration Dependence in the American Business Cycle," Journal of Political Economy, 98(3), 596-616.

Diebold, F. X., G. D. Rudebusch, and D. E. Sichel (1991): "Further evidence on business cycle duration dependence," Discussion Paper 91-11, Federal Reserve Bank of Philadelphia.

Engle, R. F., and C. W. J. Granger (1987): "Co-integration and Error Correction: Representation, Estimation, and Testing," Econometrica, 55(2), $251-76$.

Everts, M. P. (2006): "Band-Pass Filters," Discussion paper, University of Bern, Department of Economics.

Filardo, A. J., and S. F. Gordon (1998): "Business cycle durations," Journal of Econometrics, 85(1), 99-123.

Forni, M., M. Hallin, M. Lippi, and L. Reichlin (2000): "The Generalized Dynamic-Factor Model: Identification And Estimation," The Review of Economics and Statistics, 82(4), 540-554.

Hamilton, J. D. (1989): "A New Approach to the Economic Analysis of Nonstationary Time Series and the Business Cycle," Econometrica, 57(2), 357-84. 
HARDing, D., And A. PAGAN (2002): "Dissecting the cycle: a methodological investigation," Journal of Monetary Economics, 49(2), 365-381.

- (2003): "A comparison of two business cycle dating methods," Journal of Economic Dynamics and Control, 27(9), 1681-1690.

Harvey, A. C. (1985): "Trends and Cycles in Macroeconomic Time Series," Journal of Business 83 Economic Statistics, 3(3), 216-27.

InklaAR, R., J. Jacobs, and W. Romp (2003): "Business cycle indexes: Does a heap of data help?," Discussion Paper 200312, University of Groningen, CCSO Centre for Economic Research.

Kendall, M. G., and A. Stuart (1966): The Advanced Theory of Statistics: Design and Analysis, and Time-Series, vol. 3. Charles Griffin and Company, London.

King, R. G., And C. I. Plosser (1994): "Real business cycles and the test of the Adelmans," Journal of Monetary Economics, 33(2), 405-438.

King, R. G., and S. T. Rebelo (1993): "Low frequency filtering and real business cycles," Journal of Economic Dynamics and Control, 17(1-2), 207231.

Mitchell, W. C. (1927): Business Cycles: The Problem and Its Setting. National Bureau of Economic Research, New York.

Nelson, C. R., and C. I. Plosser (1982): "Trends and Random Walks in Macroeconomic Time Series: Some Evidence and Implications," Journal of Monetary Economics, 10(2), 139-62.

Pedersen, T. M. (1998): "How Long are Business Cycles? Reconsidering Fluctuations and Growth," Discussion Paper 98-24, University of Copenhagen, Institute of Economics.

Prescott, E. C. (1986): "Theory ahead of business cycle measurement," Quarterly Review, (Fall), 9-22. 
Stock, J. H., and M. W. Watson (1998): "Business Cycle Fluctuations in U.S. Macroeconomic Time Series,” Discussion Paper 6528, National Bureau of Economic Research, Inc.

United Nations (2003): Natinal Accounts Statistics: Main aggregates and detailed tables, 2001. United Nations, New York.

Watson, M. W. (1986): "Univariate Detrending Methods with Stochastic Trends," Journal of Monetary Economics, 18(1), 49-75.

(1994): "Business-Cycle Durations and Postwar Stabilization of the U.S. Economy," American Economic Review, 84(1), 24-46. 